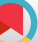

\title{
Psychometric Evaluation of the Persian Version of Dietary Habits Questionnaire and Nutrition Knowledge Questionnaire for Adults
} Among Medical Staffs

\author{
Ehsan Eshaghi ${ }^{1}$, Fatemeh Rahmati-Najarkolaei ${ }^{2,{ }^{*}}$, Mirsaeed Yekaninejad ${ }^{3}$ and Hosein Rostami ${ }^{2}$ \\ ${ }^{1}$ Department of Basic Sciences, The Education Complex of Agriculture and Animal Science of Torbat-e-Jam, Torbat-e-Jam, Iran \\ ${ }^{2}$ Health Research Center, Life Style Institute, Baqiyatallah University of Medical Sciences, Tehran, Iran \\ ${ }^{3}$ Department of Epidemiology and Biostatistics, School of Public Health, Tehran University of Medical Sciences, Tehran, Iran \\ "Corresponding author: Health Research Center, Life Style Institute, Baqiyatallah University of Medical Sciences, Tehran, Iran. Tel: +98-2187554492, Email: \\ fatemeh_rahmati@bmsu.ac.ir
}

Received 2019 May 18; Revised 2019 August 18; Accepted 2019 August 21.

\begin{abstract}
Background: Overweight in adults has increased significantly in developed countries in recent decades. This problem has attracted interests of researchers in the field of nutrition knowledge.

Objectives: The purpose of this study is to evaluate the validity and reliability of the Persian version of the dietary habits and nutritional knowledge questionnaire and the nutrition knowledge questionnaire for adults, and to confirm their structural model in the Iranian population.

Methods: A sample of 300 hospital employees from a hospital in Mashhad, Iran, participated in this study and completed the questionnaires. The questionnaires were translated using the forward-backward translation method. Psychometric evaluation of the Persian version of the questionnaires with a total 5 subscales was assessed based on content validity and construct validity using confirmatory factor analysis. Internal consistency and test-retest reliability were evaluated using Chronbach's alpha and intra-class correlation coefficient, respectively.

Results: For qualitative content validity, the content validity index scores for all items were $\geq 0.80$ and the content validity ratio scores were $0.91,0.87,0.84,0.9$, and 0.85 for all subscales. The Persian version of the questionnaires were confirmed by calculating confirmatory factor analysis models fit indices. The internal consistency figures for the Nutrition knowledge questionnaire for adults subscales were $0.7,0.75$, and 0.73 and for the total scale was 0.78 ; for the dietary habits and nutritional knowledge questionnaire were 0.71 and 0.7 and for the total scale was 0.74 . The intra-class correlation coefficient were 0.97 and 0.95 for the total scale of nutrition knowledge questionnaire for adults and the total scale of dietary habits and nutritional knowledge questionnaire respectively.

Conclusions: The findings show that the Persian version of the questionnaires has good content validity as well as acceptable validity and reliability.
\end{abstract}

Keywords: Construct Validity, Dietary Habits and Nutrition Knowledge, Forward-Backward Translation, Psychometric Evaluation, Reliability and Validity

\section{Background}

Overweight in adults has increased significantly in developed countries in recent decades. This problem has attracted interests of researchers in the field of nutrition knowledge. Nutrition knowledge is an important factor based on which researchers are trying to develop a nutrition knowledge scale.

The nutrition knowledge questionnaires developed so far have some limitations. Either they are weak in the area of psychometric validation or they target a limited area of nutrition knowledge (1-8). For example, Towler and
Shepherd showed that their questionnaire has good internal reliability and construct validity (9). Their study does not include the kind of psychometric validation. The content validity is questionable because of little explanation on how the items were generated. That questionnaire has no questions about dietary recommendations and the relationship between disease and diet, but the questionnaire evaluates knowledge about the nutrient content of foods. Anderson et al conducted a study using a questionnaire with good content validity (10). In contrast to Towler and Shepherd's questionnaire, the items concerned famil- 
iarity with nutrition-related phrases as well as knowledge about dietary recommendations and their practical application. But the internal consistency of this questionnaire is poor, and the study was not subjected to construct validity and test-retest reliability. So we want the questionnaires that consider both aspects of psychometric validation and nutrition knowledge, as well as dietary habits. Nutrition knowledge questionnaire for adults (NKQA) (11), and the dietary habits and nutritional knowledge (DHNK) questionnaire (12) together are included nutrition knowledge and dietary habits and also consider psychometric validation. There is no overlap or similarity in nutrition knowledge questions between two questionnaires. By these two questionnaires we want to study a wide range of nutritional issues of Iranian adults for the very first time. Nutrition knowledge is important because it affects food choices. Some studies have shown that application of the beliefs models in increasing nutrition knowledge can lead to better dietary behavior and intake $(13,14)$. Over the last few decades, the people of Iran have changed their eating patterns. The rising incidence of obesity and chronic diseases may be due to an imbalance in dietary patterns (15). So far, no research has focused on the psychometric evaluation of a nutrition knowledge and dietary habit questionnaires with regard to Iranian adults.

On the other hand, scales in a foreign language can be bound to a certain a culture. We cannot use such questionnaires directly because of the existence of cultural bias. Translation is a common method to prepare questionnaires for cross-cultural adaptation. So after translation, it necessitates assessing psychometric evaluation of translated questionnaires (16-18).

Therefore, this study carries out a psychometric evaluation of a Persian version of the scales and confirms its structural model among Iranian medical staff at a hospital in Mashhad city, which is in northeast Iran. Also the researchers offer this scale for more studies to use in order to gather data about dietary habits and find ways to raise public awareness about nutrition and unhealthy dietary habits. Hereby overweight/obesity can be prevented.

\section{Objectives}

The present study aimed to evaluate the validity and reliability of the Persian version of the dietary habits and nutritional knowledge questionnaire and the nutrition knowledge questionnaire for adults, and to confirm their structural model in the Iranian population.

\section{Methods}

The scale used in this study combines two English questionnaires. Questionnaire 1 is a DHNK questionnaire that includes two subscales: dietary habits (18 items which are scored on a four-point Likert scale from 1 (never) to 4 (always)) and nutrition knowledge (29 items which are scored on a four-point Likert scale from 1 (strongly disagree) to 4 (strongly agree)). The reliability coefficients for original DHNK questionnaire (English version) were 0.66 and 0.645 for dietary habits and nutritional knowledge, respectively (12). Questionnaire 2 is NKQA, which contains three subscales: familiarity with MyPyramid (20 items), the nutrition content of foods (15 items), and the dietdisease relationship (11 items). Each item was scored either correct or incorrect, and the scores were then totalled for each subscale and the questionnaire overall. For original English version of NKQA Cronbach's alpha and test-rest were 0.91 and 0.95 respectively which demonstrating internal consistency reliability and test-retest reliability (11). There are two sections in the combined questionnaire: (1) demographic variables such as gender, age, academic degree, and body mass index, and (2) the main part, which consists of 93 statements ( 46 items for NKQA and 47 items for DHNK) on nutritional information and dietary habits.

The research design consists of a translation procedure and psychometric evaluation. This includes assessing content validity, construct validity, and reliability. Permission for translation of the NKQA and DHNK questionnaires was obtained by email from Prof. Anna Marie Jones and Prof. Carol Biddington, respectively. The original English version of NKQA and DHNK questionnaire were disseminated in years 2015 and 2005 respectively $(11,12)$. A total of 332 questionnaires were distributed in the second half of 2016. 12 respondents were excluded following the exclusion criteria and 20 respondents denied to end up with the 300. The staff inclusion criteria were being able to read and write Persian, and being over 18 years of age.

\subsection{Procedure}

The NKQA and DHNK questionnaires were translated into Persian by using the Brislin translation method (forward translation)(19). Two experts of health education and nutrition who were familiar with English and Persian did the translation work. They translated the questionnaires to Persian independently. The two translations were then formed into a single unit. Thereafter, it was sent to health education and nutrition experts to evaluate its content validity. The translated version was also provided to the other experts of health education and nutrition for their comments. The goal was to ensure that the items of the questionnaires had no ambiguity, and that different experts had the same understanding of the items. The final version of the translated scale was approved using the most appropriate translated terms and the imperfections were removed. Then, an English language expert who was unfamiliar with the content of the primary English question- 
naires performed backward translation from Persian to English. The English language expert provided his comments and, by comparing the English questionnaires, the necessary changes were applied to the Persian translation. This version of the questionnaires was then distributed among 10 experts in the field of nutrition knowledge to evaluate its content validity.

\subsubsection{Content Validity}

To assess the content validity index (CVI), the experts were asked to talk about each item in order to assess its simplicity, relevancy, and clarity with regard to the content as per the Waltz and Bausell index (20). The experts also were asked to rate scale items on a 4-point ordinal scale (Table 1) (14).

\begin{tabular}{l|c}
\hline \multirow{2}{*}{ Table 1. Scoring Method } & Details \\
\hline \multirow{4}{*}{ Simplicity } & not simple \\
\cline { 2 - 2 } & item need some revision \\
\cline { 2 - 2 } & simple but need minor revision \\
\cline { 2 - 2 } Relevancy & very simple \\
\cline { 2 - 2 } & not relevant \\
\cline { 2 - 2 } & item need some revision \\
\cline { 2 - 2 } & relevant but need minor revision \\
\hline \multirow{4}{*}{ Clarity } & very relevant \\
\cline { 2 - 2 } & not clear \\
\cline { 2 - 2 } & item need some revision \\
\cline { 2 - 2 } & clear but need minor revision \\
\hline
\end{tabular}

To obtain content validity index for simplicity, relevancy and clarity of each item, the number of those judging the item as simple or relevant or clear (rating 3 or 4 ) was divided by the number of content experts. The values for the CVI which are given greater or equal than 0.79 were considered acceptable (0.7 - 0.78: revision and correction; $<0.70$ : unacceptable) (20).

The experts were also requested to assess each item as 'essential', 'useful but not essential', or 'not necessary' in order to compute the content validity ratio (CVR). A decision was made to accept or reject CVR values using the Lawshe table (21). This table shows the critical CVR values for different values of the sample size of experts. A test evaluator could determine the size of the calculated CVR that is necessary to exceed the chance expectation. Lawshe developed following formula to calculate content validity ratio.

$\mathrm{CVR}=\left(\mathrm{n}_{\mathrm{e}}-\mathrm{N} / 2\right) /(\mathrm{N} / 2)$

Where $n_{e}$ is the number of experts assessed the item as essential and $\mathrm{N}$ is total number of experts.
In addition to determine the quality of the content validity, floor and ceiling effects were computed. A floor or ceiling effects of $15 \%$ or less is considered acceptable (22).

\subsubsection{Reliability}

The reliability of the Persian version of the questionnaires was first assessed through evaluating internal consistency. Cronbach's alpha coefficient was employed to assess the internal consistency of the questionnaires. If the value of Cronbach's alpha coefficient is greater than or equal to 0.7 , it indicates satisfactory internal consistency (23). After that, the construct reliability (CR) of each of the subscales was evaluated (24). CR values between 0.6 and 0.7 can be accepted provided other indicators are good (25). In addition, to compute the intra-class correlation coefficient (ICC), and the stability and reliability through the test-retest, the questionnaire was given to 30 health employees in two-week intervals. The ICC values which are greater or equal than 0.60 were considered satisfactory $(\mathrm{r}$ $\geq 0.81-1.0$ as excellent, $0.61-0.80$ very good, $0.41-0.60$ good, 0.21 - 0.40 fair, and 0.0 - 0.20 poor) (26). Data were analysed using SPSS version 23.

\subsubsection{Construct Validity}

To determine construct validity, confirmatory factor analysis (CFA) was performed. The LISREL program version 8.80 was used to carry out CFA in order to ensure that the factor structure of the translated version of the questionnaires is similar to the original version. LISREL is a program that can perform CFA to release model fit indices (27). The values are shown on the side of the box in LISREL outputs (path diagrams) are errors (Figures 1 and 2). In our study, as errors decrease, the factor loadings will increase.

We used following fit indices in the present study and their acceptable ranges are reported as following $(26,28$, 29): the chi-squared goodness-of-fit test (chi-square/df) $<5$ (<3: good), root mean square error of approximation (RMSEA) < 0.1 (< 0.08: good), The normed fit index (NFI) $>0.9$ as well as non-normed fit index (NNFI) $>0.9$ and comparative fit index $(\mathrm{CFI})>0.9$.

\subsubsection{Sample Size}

Determining the sample size is a prerequisite in order to perform CFA. There are several recommendations for an acceptable sample size for factor analysis. Some authors recommend 5 persons per item while others recommend $10(30,31)$. The original questionnaires used in this study have 46 and 47 items. So, the recommended samples for each individual questionnaire ranged from 230 to 470. To get the samples for factor analysis, stratified sampling was used. The hospital staff was classified into physicians, nurses, and other employees. All participants gave 

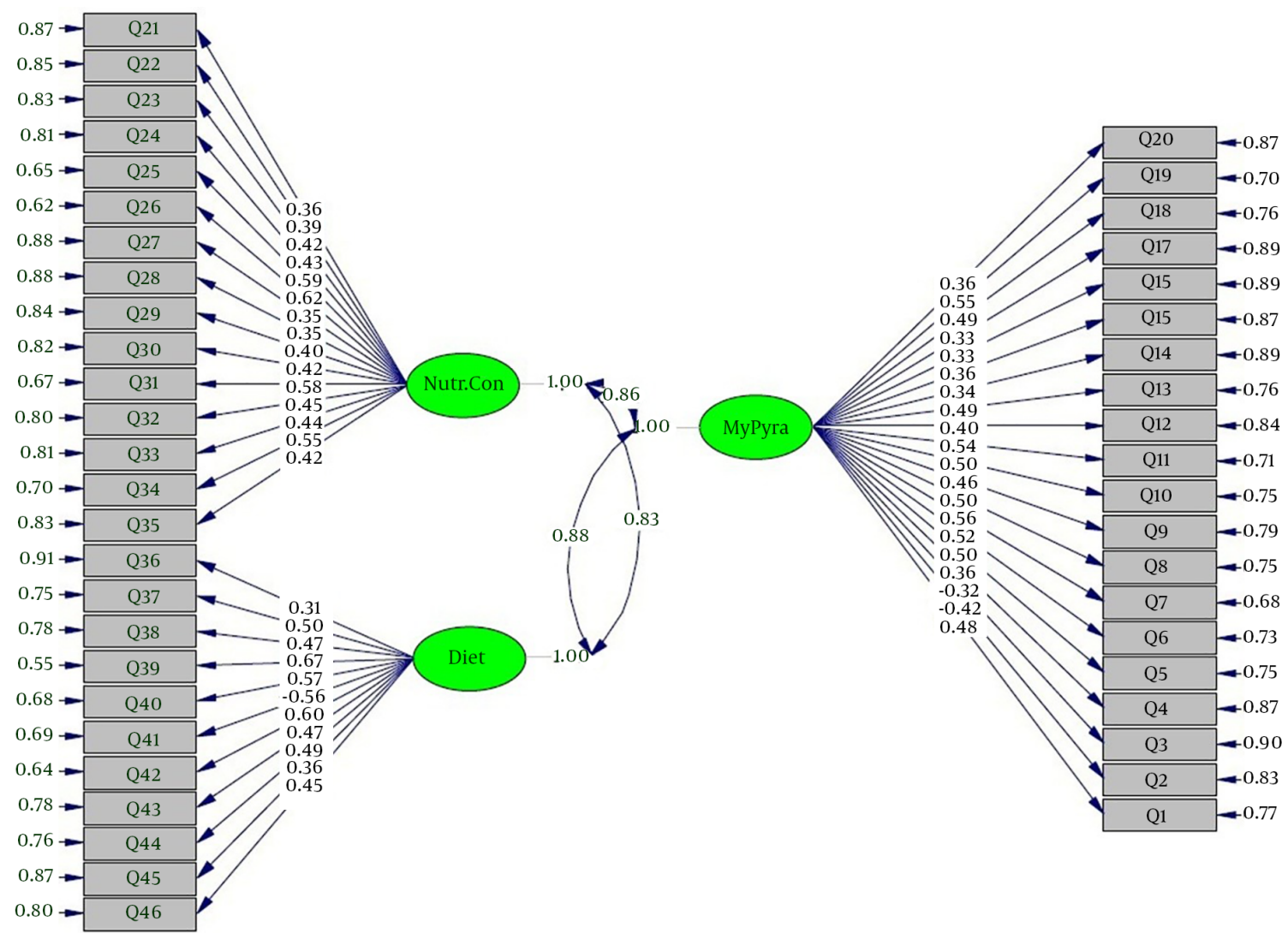

Figure 1. Confirmatory factor structure of NKQA

informed consent according to the research ethic committee of the Baqiyatallah University of Medical Sciences. Strata sample sizes are determined by the following equation:

$$
\mathrm{n}_{\mathrm{h}}=\left(\mathrm{N}_{\mathrm{h}} / \mathrm{N}\right) * \mathrm{n}
$$

where $n_{h}, N_{h}, N$ and $n$ denote the sample size for stratum $\mathrm{h}$, the population size for stratum $\mathrm{h}$, total population size and total sample size respectively. For example for physician stratum, we obtained $\left(\mathrm{N}_{\mathrm{h}} / \mathrm{N}\right)=0.04$. So the sample size for this stratum is $300 * 0.04$.

\section{Results}

The participants comprised physicians, nurses, and other employees working at a hospital in Mashhad. Thereby, the content validity, internal consistency, and construct validity of the Persian version of the two questionnaires was obtained. The mean ages of the participants were $30.7 \pm 8.3$ years. Of the 300 respondents, $41.8 \%$ were male and $58.2 \%$ were married. The demographic characteristics of the study sample are presented in Table 2.

\begin{tabular}{|l|l|}
\hline \multicolumn{1}{|l|}{ Table 2. Demographic Characteristics of Sample } & \\
\hline Variable & Values $^{\mathbf{a}}$ \\
\hline Gender & $125(41.7)$ \\
\hline Male & $175(58.3)$ \\
\hline Female & \\
\hline Job title & $12(4)$ \\
\hline Physician & $93(31)$ \\
\hline Nurse & $195(65)$ \\
\hline Other employees & \\
\hline Academic degree & $14(4.7)$ \\
\hline PhD & $45(15)$ \\
\hline MSc & $156(52)$ \\
\hline BSc & $38(12.7)$ \\
\hline Associate Degree & $37(12.3)$ \\
\hline High school diploma & $10(3.3)$ \\
\hline Less than Diploma & $23.74 \pm 3.6$ \\
\hline BMI (body mass index) & \\
\hline
\end{tabular}

${ }^{a}$ Values are expressed as frequency (\%) or mean $\pm S D$.

The results of reliability of test-retest and internal consistency are reported in Table 3. 


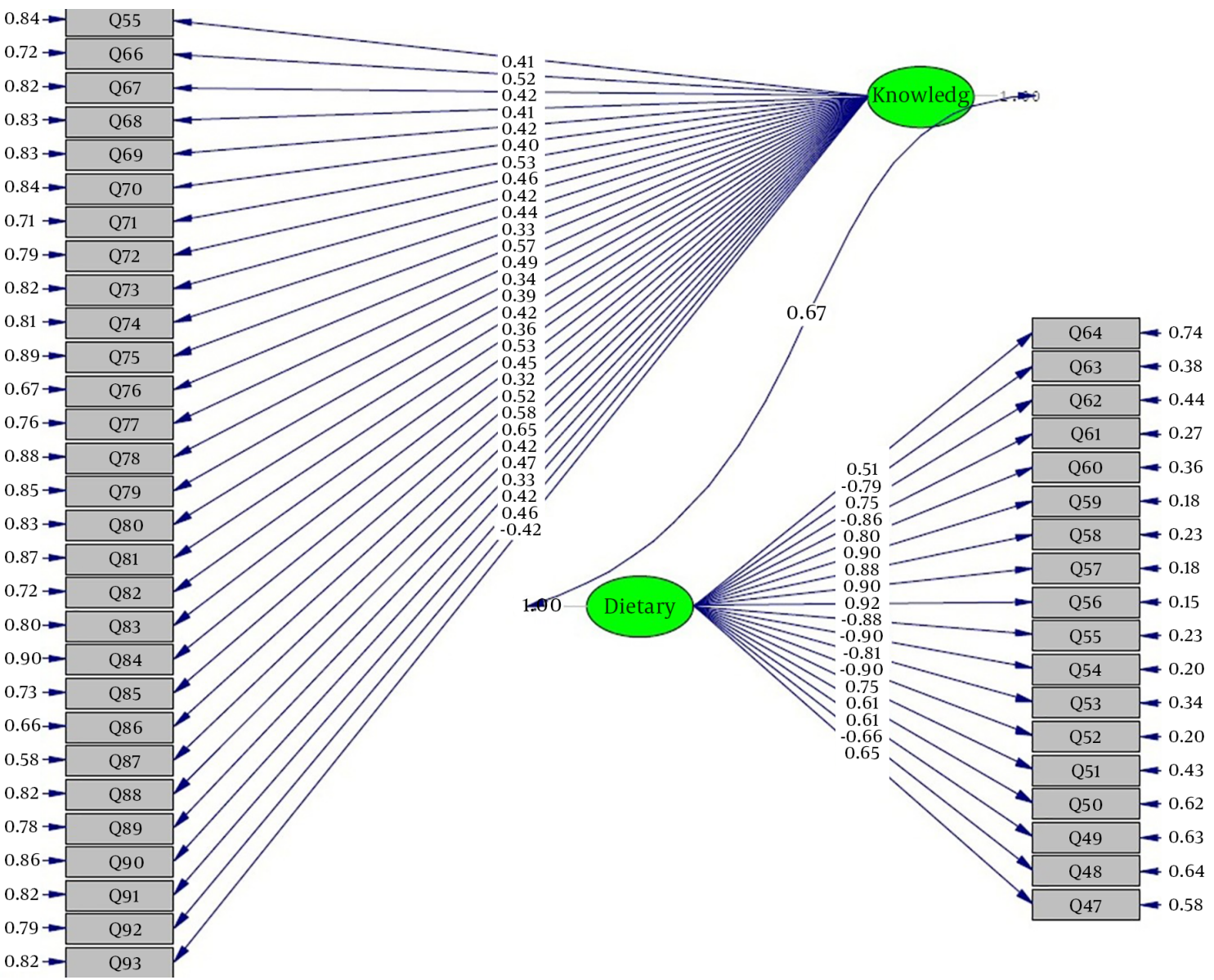

Figure 2. Confirmatory factor structure of DHNK

\begin{tabular}{|c|c|c|c|c|c|c|c|c|}
\hline Scales (Range) & Subscale & No. of Items & $\mathbf{r}$ & CR & Mean & SD & Floor Effect (\%) & Ceiling Effect (\%) \\
\hline NKQA (0-22) & Familiarity with MyPyramid & 20 & 0.95 & 0.83 & 9.3 & 3.71 & 0.3 & 0.3 \\
\hline NKQA (0-26) & Nutrition content of foods & 15 & 0.97 & 0.80 & 11.93 & 5.06 & 1.7 & 0.3 \\
\hline NKQA (0-18) & Diet-disease relationship & 11 & 0.98 & 0.78 & 8.7 & 3.92 & 0.3 & 6 \\
\hline DHNK (18-72) & Dietary habits & 18 & 0.99 & 0.96 & 51 & 4.46 & 0.3 & 1 \\
\hline DHNK (29-116) & Nutritional knowledge & 29 & 0.94 & 0.88 & 84.76 & 7.93 & 0.3 & 0.3 \\
\hline
\end{tabular}

Abbreviations: CR, construct reliability; r, test-retest reliability coefficient (ICC)

The Cronbach's alpha coefficients for the total scale of NKQA and DHNK were 0.78 and 0.74 respectively. Also the ICC for the total scale of NKQA was 0.97 and for DHNK was 0.95. Table 3 also shows participants mean scores. High scores in the following subscales show good nutrition knowledge of participants: familiarity with MyPyramid, nutrition content of foods, diet-disease relationship and nutritional knowledge. High dietary habits subscale scores reveal healthy participants' dietary habits.

In this study, 10 experts provided comments. They found the questionnaires satisfactory and approved all items in it. The CVR scores were 0.91, 0.87, 0.84, 0.9, and 0.85 for the subscales of familiarity with MyPyramid, nutrition content of foods, diet-disease relationship, dietary habits, and nutrition knowledge, respectively. Besides, the CVI scores for all items were $\geq 0.80$ and the qualitative 
content validity was approved. The mean CVI for all items was $89 \%$ for relevancy, $87 \%$ for clarity, and $90 \%$ for simplicity. Floor and ceiling effects of all subscales are in acceptable range.

To determine construct validity, CFA was conducted separately for the two translated questionnaires. Table 4 shows the results of the CFA on the Persian versions of the NKQA and DHNK questionnaires.

The CFA results showed that the translated Persian version of the questionnaires was good enough to fit. All indices are in an acceptable range and show strong goodness of fit. Figures 1 and 2 show the confirmatory factor structure of NKQA and DHNK. Saggino and Kline suggested that items with absolute loading values of 0.3 or greater should be considered appropriate (32). In our study, all factor loadings are greater than 0.3. The negative factor loadings mean that hidden factors have the characteristic opposite of whatever the observable measures measure.

The values of construct reliability for all subscales are greater than 0.7 and show the reliability of the translated scale. In addition, the ICC values are greater than 0.81 and satisfactory. With regard to the CVI and CVR values, the Persian version of the scale has good content validity. As a reliable and valid scale, it can be used among Iranian adults.

\section{Discussion}

There is a relationship between the internal consistency and homogeneity of the scale (33). In well-designed scales, Cronbach's alpha coefficient is greater than 0.7 (34). Kliemann et al. (35) evaluated an updated version of the general nutrition knowledge questionnaire (GNKQ). They reported that the reliability was greater than 0.7 for all sections, and their updated scale was reliable and valid. Then the values of construct reliability for all subscales were greater than 0.7 which indicated good reliability (24). In another study on nutrition involving a 20-item questionnaire on nutrition knowledge, internal consistency reliability and test-retest reliability were 0.74 and 0.86 . This revealed the validity and reliability of the scale (36). For the present study, the level of internal consistency is satisfactory. To investigate the stability, the ICC was used and the responses showed high stability. All subscales had good internal consistency and test-retest reliability.

The studies that assessed original questionnaires, did not fit CFA models to questionnaires $(11,12)$. In this paper CFA models fitted to the translated questionnaires and all fit indices were in acceptance range.

However, the scale was used among health employees, but it does not mean that the scale is not applicable to the general population. Because according to Table $2,65 \%$ of staff has no health-related field of study. Also $28.3 \%$ of staff has no any college education.
The strength of this study is that it has completed the psychometric process to provide a valid and reliable scale for the first time in Iran. The scale needs to be used by experts to assess nutritional knowledge and dietary habits of general population.

One of the limitations of our study is that, although the scale was demonstrated to be valid and reliable, the large number of items may make it boring and some participants may not complete it. Regarding to Table 3, by the time the participants start the second questionnaire (NKQA), they are tired and the scores drop. But they have better scores on items relating to dietary habits and nutrition knowledge (DHNK). The questionnaires order effect bias can be minimised by changing the order the questionnaires are administered. Moreover, the mean of the employees' BMI staff shows that the employees are healthy, which may be due to healthy habits. A decline in the scores of the NKQA subscales could be due to the large number of questions. A shorter version of this scale could give better results because a shorter version would have reduced the variation in the answers. The questionnaire's items can be reduced by using higher cutoff for loading factor, or using data reduction analyses such as "component factor analysis". It is an important issue tough what items would be excluded to shorten the tool and what loss would be had in the information collected. But in this study, the aim is to evaluate the validity and reliability of the Persian version of the original questionnaires with all items. Whether and how this subject can be adapted to our scale is an interesting project for further investigations.

\subsection{Conclusions}

This reliable and valid scale can be used in Iranian population.

\section{Acknowledgments}

This paper originated from Health Research Center of Baqiyatallah University of Medical Sciences. Authors thank all medical staffs and managers of the selected hospital for cooperation.

\section{Footnotes}

Authors' Contribution: Study concept and design: Ehsan Eshaghi and Fatemeh Rahmati-Najarkolaei. Analysis and interpretation of data: Ehsan Eshaghi. Drafting of the manuscript: Ehsan Eshaghi. Critical revision of the manuscript for important intellectual content: Mirsaeed Yekaninejad, Hosein Rostami and Fatemeh RahmatiNajarkolaei. Statistical analysis: Ehsan Eshaghi.

Conflict of Interests: The authors declare that they have no conflicts of interest. 


\begin{tabular}{|c|c|c|c|c|c|c|c|}
\hline Goodness of Fit Indices & Chi-Square & Df & Chi-Square/df & CFI & NFI & NNFI & RMSEA \\
\hline NKQA & 1977.09 & 973 & 2.03 & 0.92 & 0.86 & 0.92 & 0.059 \\
\hline DHNK & 1890.16 & 998 & 1.89 & 0.97 & 0.93 & 0.96 & 0.055 \\
\hline
\end{tabular}

Ethical Approval: The study gets ethical approval by the Research Committee of the Baqiyatallah University of Medical Sciences, Tehran, Iran, (MHRc 540).

Funding/Support: This research received no specific grant from any funding agency, commercial or not-forprofit sectors.

\section{References}

1. Furber MJW, Roberts JD, Roberts MG. A valid and reliable nutrition knowledge questionnaire for track and field athletes. BMC Nutr. 2017;3(1). doi: 10.1186/s40795-017-0156-0.

2. Dumic A, Miskulin M, Pavlovic N, Orkic Z, Bilic-Kirin V, Miskulin I. The nutrition knowledge of Croatian general practitioners.JClin Med. 2018;7(7). doi: 10.3390/jcm7070178. [PubMed: 30029463]. [PubMed Central: PMC6068600].

3. Spendlove JK, Heaney SE, Gifford JA, Prvan T, Denyer GS, O'Connor HT. Evaluation of general nutrition knowledge in elite Australian athletes. BrJ Nutr.2012;107(12):1871-80. doi: 10.1017/S0007114511005125. [PubMed: 22018024].

4. Anderson AS, Bell A, Adamson A, Moynihan P. A questionnaire assessment of nutrition knowledge-validity and reliability issues. Public Health Nutr. 2002;5(3):497-503. doi: 10.1079/PHNPHN2001307. [PubMed: 12003663].

5. Sami W, Ansari T, Butt NS, Ab Hamid MR. Psychometric evaluation of dietary habits questionnaire for type 2 diabetes mellitus. J Phys Conf Ser. 2017;890:12151. doi: 10.1088/1742-6596/890/1/012151.

6. De Souza Silveira R, Kratzenstein S, Hain G, Mayer F, Carlsohn A. Nutrition knowledge quiestionnaire - modified and validated for the use in German adolescent athletes. German J Sports Med.2015;2015(9):24852. doi: $10.5960 /$ dzsm.2015.190.

7. Bradette-Laplante M, Carbonneau E, Provencher V, Begin C, Robitaille J, Desroches S, et al. Development and validation of a nutrition knowledge questionnaire for a Canadian population. Public Health Nutr. 2017;20(7):1184-92. doi: 10.1017/S1368980016003372. [PubMed: 28025953].

8. Zinn C, Schofield G, Wall C. Development of a psychometrically valid and reliable sports nutrition knowledge questionnaire. J Sci Med Sport.2005;8(3):346-51. doi:10.1016/s1440-2440(05)80045-3. [PubMed: 16248475].

9. Towler G, Shepherd R. Development of a nutritional knowledge questionnaire. J Hum Nutr Diet. 1990;3(4):255-64. doi: 10.1111/j.1365277X.1990.tb00065.x.

10. Anderson AS, Umapathy D, Palumbo L, Pearson DWM. Nutrition knowledge assessed by a structured questionnaire in a group of medical in-patients. J Hum Nutr Diet. 1988;1(1):39-46. doi: 10.1111/j.1365277X.1988.tb00262.x.

11. Jones AM, Lamp C, Neelon M, Nicholson Y, Schneider C, Wooten Swanson P, et al. Reliability and validity of nutrition knowledge questionnaire for adults. J Nutr Educ Behav. 2015;47(1):69-74. doi: 10.1016/j.jneb.2014.08.003. [PubMed: 25242506].

12. Paugh SL. Dietary habits and nutritional knowledge of college athletes [dissertation]. California University of Pennsylvania; 2005.

13. Ford JC, Pope JF, Hunt AE, Gerald B. The effect of diet education on the laboratory values and knowledge of hemodialysis pa- tients with hyperphosphatemia. J Ren Nutr. 2004;14(1):36-44. doi: 10.1053/j.jrn.2003.09.008. [PubMed: 14740329].

14. Wardle J, Parmenter K, Waller J. Nutrition knowledge and food intake. Appetite. 2000;34(3):269-75. doi: 10.1006/appe.1999.0311. [PubMed: 10888290].

15. Esmaili H, Bahreynian M, Qorbani M, Motlagh ME, Ardalan G, Heshmat R, et al. Prevalence of general and abdominal obesity in a nationally representative sample of Iranian children and adolescents: The CASPIAN-IV study. Iran J Pediatr. 2015;25(3). e401. doi: 10.5812/ijp.25(3)2015.401. [PubMed: 26199707]. [PubMed Central: PMC4505989].

16. Moser AM, Traebert]. [Transcultural adaptation of the HIV/AIDS-target quality of life for HIV/AIDS for the evaluation of patients' quality of life]. Cien Saude Colet. 2011;16 Suppl 1:1357-62. Portuguese. doi: 10.1590/s1413-81232011000700070. [PubMed: 21503486].

17. Rode N. Translation of measurement instruments and their reliability: An example of job-related affective well-being scale. Metodoloski Zvezki. 2005;2(1):15.

18. Carrico G, Meves R, Avanzi O. Cross-cultural adaptation and validity of an adapted Brazilian Portuguese version of Scoliosis Research Society-30 questionnaire. Spine (Phila Pa 1976). 2012;37(1):E60-3. doi: 10.1097/BRS.0b013e31823c7cd6. [PubMed: 22045004].

19. Brislin RW. Back-translation for cross-cultural research. J Cross Cult Psychol.2016;1(3):185-216. doi: 10.1177/135910457000100301.

20. Waltz CF, Bausell BR. Nursing research: Design statistics and computer analysis. Davis FA; 1981.

21. Ayre C, Scally AJ. Critical values for Lawshe's content validity ratio. Meas Eval Counsel Dev. 2017;47(1):79-86. doi: 10.1177/0748175613513808.

22. Terwee CB, Bot SD, de Boer MR, van der Windt DA, Knol DL, Dekker J, et al. Quality criteria were proposed for measurement properties of health status questionnaires. J Clin Epidemiol. 2007;60(1):34-42. doi: 10.1016/j.jclinepi.2006.03.012. [PubMed: 17161752].

23. DeVon HA, Block ME, Moyle-Wright P, Ernst DM, Hayden SJ, Lazzara DJ, et al. A psychometric toolbox for testing validity and reliability. $J$ Nurs Scholarsh. 2007;39(2):155-64. doi: 10.1111/j.1547-5069.2007.00161.X. [PubMed: 17535316].

24. Hair JF, Black WC, Babin BJ, Anderson RE, Tatham RL. Multivariate data analysis. 6. Upper Saddle River, NJ: Pearson Prentice Hall; 2006.

25. Kline RB. Structural equation modeling. New York: Guilford Press; 1998. $427 \mathrm{p}$.

26. Munro BH. Statistical methods for health care research. Lippincott Williams \& Wilkins; 2005

27. Diamantopoulos A, Siguaw JA. Introducing LISREL: A guide for the uninitiated. Sage; 2013.

28. Schreiber JB, Nora A, Stage FK, Barlow EA, King J. Reporting structural equation modeling and confirmatory factor analysis results: A review.J Educ Res. 2006;99(6):323-38. doi: 10.3200/joer.99.6.323-338.

29. Hooper D, Coughlan J, Mullen MR. Structural equation modelling: Guidelines for determining model fit. Electronic J Bus Res Methods. 2008;6(1):53-60.

30. Worthington RL, Whittaker TA. Scale development research. Counsel Psychol. 2016;34(6):806-38. doi: 10.1177/0011000006288127.

31. Rattray J, Jones MC. Essential elements of questionnaire design and development. J Clin Nurs. 2007;16(2):234-43. doi: 10.1111/j.13652702.2006.01573.x. [PubMed: 17239058].

32. Saggino A, Kline P. Item factor analysis of the italian version of the death anxiety scale. J Clin Psychol. 1996;52(3):329-33. doi: 10.1002/(SICI)1097-4679(199605)52:3<329::AID-JCLP11>3.0.CO;2-K. [PubMed: 8835696]. 
33. Streiner DL, Norman GR. Health measurement scales: A practical guide to their development and use. Oxford Medical Publications; 2003.

34. van Griethuijsen RALF, van Eijck MW, Haste H, den Brok PJ, Skinner $\mathrm{NC}$, Mansour N, et al. Global patterns in students' views of science and interest in science. Res Sci Educ. 2014;45(4):581-603. doi:10.1007/s11165014-9438-6.

35. Kliemann N, Wardle J, Johnson F, Croker H. Reliability and validity of a revised version of the general nutrition knowledge questionnaire. EurJClin Nutr. 2016;70(10):1174-80. doi:10.1038/ejcn.2016.87. [PubMed: 27245211]. [PubMed Central: PMC5014128].

36. Tabbakh T, Freeland Graves J. Healthy eating attitude: A mediator of nutrition knowledge and diet quality using the healthy eating index-2010 in young women. Journal of Womens Health, Issues and Care. 2016;5(2). doi:10.4172/2325-9795.1000226. 